\title{
Minimal Semantics and Word Sense Disambiguation
}

\author{
Luca Gasparri \\ Institut Jean Nicod - ENS Paris \\ BIBLID [0873-626X (2014) 39; pp. 147-171]
}

DOI: $10.2478 /$ disp-2014-0011

\begin{abstract}
Emma Borg has defined semantic minimalism as the thesis that the literal content of well-formed declarative sentences is truth-evaluable, fully determined by their lexico-syntactic features, and recoverable by language users with no need to access non-linguistic information. The task of this article is threefold. First, I shall raise a criticism to Borg's minimalism based on how speakers disambiguate homonymy. Second, I will explore some ways Borg might respond to my argument and maintain that none of them offers a conclusive reply to my case. Third, I shall suggest that in order for Borg's minimalism to best accommodate the problem discussed in this paper, it should allow for semantically incomplete content and be converted into a claim about linguistic competence.
\end{abstract}

\section{Keywords}

Semantic minimalism, lexico-syntactic processing, literal meaning, word sense disambiguation, homonymy.

\section{Introduction}

Emma Borg (2004, 2012) has characterized semantic minimalism as the natural inheritor of a formal semantics approach to sentential meaning and has defended the idea of a purely lexico-syntactic route to propositional content. In her view, literal content for wellformed declarative sentences is truth-evaluable, fully determined by their lexico-syntactic features, and recoverable by language users with no need to access contextual information or world knowledge. Sentences have their truth-conditional content determined independently of non-linguistic factors, and the contribution of context to the recovery of sentential meaning is limited to the saturation of a

Disputatio, Vol. VI, No. 39, November 2014

Received: 15/12/2013 Revised: 09/02/2014 Accepted: 28/07/2014 
narrow class of indexical expressions. Semantic minimalism thus opposes contextualist, relativist and occasion-sensitive views maintaining that the bearers of propositional content are utterances, rejects the all-pervasive constructive role for non-linguistic context envisaged by dual pragmatics, proposes that the proper task of semantic theories is to account for the literal meaning of sentences rather than for the communicated content of speech acts, and is committed to an orthodox view of compositionality, according to which, barring explicit indexicals, the truth-evaluable content of sentential expressions is entirely a function of the combination of their syntactic architecture with the stable semantic input of their lexical constituents. ${ }^{1}$

Understood along these lines, semantic minimalism is vulnerable to two fundamental arguments (Borg 2012: 48-49):

(i) Minimal propositions are explanatorily inert: literal truth-evaluable content plays no indispensable role in (accounting for) the cognitive processes whereby speakers assign pragmatic meaning to declarative sentences;

(ii) Minimal propositions are impossible: some declarative sentences fail to convey (or encode) literal truth-evaluable content thanks to their lexico-syntactic elements alone.

To start, let us focus on argument (i). The bulk of the objection (a clear formulation of which can be found, e.g., in Recanati 2004: 1822 ) is that the entertainment of literal truth-evaluable content needs not be always included in the series of mental processes whereby speakers recover speech act content or pragmatically enriched meaning. If on a hot summer night I tell my thirsty friend John 'There is beer in the fridge', it seems there is no need for him to consciously or unconsciously entertain the literal proposition THERE IS BEER IN THE FRIDGE in order to understand that the beer I am talking about is presumably contained in cans or bottles, rather than spilled everywhere in the fridge. In other words, John needs not entertain overt quantification to determine intended quantification: he can get straight to

\footnotetext{
${ }^{1}$ For an overview of the main tenets of Borg's minimalism, see Borg 2007, 2009. Korta, Perry 2006, Jaszczolt 2007 and Stojanovic 2008 are equally useful introductions to the positions surrounding the debate on semantic minimalism.
} 
intended quantification, with no intermediate literal stops. Something similar appears to happen in the spontaneous enrichment of sentences with unarticulated content (e.g., 'The baby cried and the mother [ + OF THE BABY] picked it up'), in the recovery of the intended meaning of sentences requiring context-driven quantifier domain restrictions (e.g., 'There is no one at work, everyone went to the party'), in the interpretation of adjectives whose conventional semantic value is prima facie unable to function as a propositional constituent prior to contextual input (e.g., 'Paul is ready': for what?), in the comprehension of sentences containing presuppositions that fail to be accommodated by the context of utterance (e.g., 'The dog is thirsty' when no salient dog can be identified), ${ }^{2}$ or in the evaluation of sentences with gradable predicates (e.g., 'Mary is tall'). ${ }^{3}$ Since, the argument goes, these cases prove that the entertainment of literal truth conditions is sometimes unnecessary to determine pragmatically enriched content, semantic minimalism is wrong in requiring the composition of literal truth conditions to take place even in cases where minimal propositions make no contribution to the recovery of pragmatic meaning.

The rationale of this paper is that Borg's minimalism is susceptible to the same variety of objection even if we focus on the determination of literal sentential meaning itself, and that there is no need to

${ }^{2}$ As the informed reader will know, Frege and Strawson proposed that in similar cases the sentence fails to result in a logical form capable of having a truth value. This view of presuppositions is well-established in linguistics: see Heim 1983, Van der Sandt 1992, Beaver 2001.

${ }^{3}$ In this case, the argument from the contextualist side runs as follows. Propositions have truth values relative to circumstances of evaluation. If circumstances of evaluation are possible worlds, then propositions have truth values relative to worlds (i.e., intensions). So if there is a minimal proposition literally expressed by every utterance of 'Mary is tall' at every context of use, 'Mary is tall' must have an intension. At this point, contextualists conclude modo tollente that since the gradable adjective tall makes it impossible for 'Mary is tall' to have an intension in the standard sense of the term, there is no stable proposition literally expressed at every utterance of 'Mary is tall'. For an attempt to address the problem in a synthesis of semantic minimalism and radical contextualism, see the non-indexical contextualism of MacFarlane 2007, 2009. See Davis 2013 for a fresh discussion of MacFarlane's proposal. More on the semantics of gradable adjectives in, e.g., Kennedy 2007. 
point at the interplay of literal truth conditions and pragmatically enriched meaning to maintain that minimalism fails to match our best assumptions about the role of truth-evaluable content in meaning recovery. Bearing this in mind, my type (i) aim will be to argue that there are well-formed declarative sentences such that they cannot be assigned a literal truth-evaluable content through lexico-syntactic processing alone. This will lead me to the contention that there are cases of sentential meaning construction where the recovery of truth-evaluable content cannot be based exclusively on linguistic knowledge and, in turn, to the type (ii) claim that sentences whose literal meaning can be determined only via an interactive procedure of the above kind fail to convey a literal truth-evaluable content unless they are processed on the basis of information exceeding the boundaries of linguistic knowledge (\$2). I shall explore some ways the minimalist might respond to this objection and argue that none of them offers a conclusive reply to my case $(\S \S 3,4)$. Finally, I will suggest that Borg's minimalism should allow for semantically incomplete content and be converted from a thesis about lexico-syntactic performance into a claim about lexico-syntactic competence (\$5).

\section{Homonymy}

Consider the sentence 'A pupil was in the middle of the classroom'. Due to the presence of classroom, speakers exposed to this sentence preferentially interpret the homonymous word pupil as conveying the sense YOUNG STUDENT, despite the fact that pupil can also be taken to mean EYE OPENING. Accordingly, they tend to perceive 'A pupil was in the middle of the classroom' as a semantically definite expression despite the ambiguity of pupil. They might perceive pupil as ambiguous in the earliest stages of the speech input, when they have not yet been provided with any clue as to how pupil should be disambiguated. But as soon as they get to hear classroom, they spontaneously select YOUNG STUDENT as the most plausible sense to be assigned to pupil. The whole process runs plausibly (and very roughly) as follows. ${ }^{4}$ First,

\footnotetext{
${ }^{4}$ For the relevant empirical research, see any recent handbook of psycholinguistics with a good section on lexical processing (e.g., Traxler 2011, Spivey, McRae, Joanisse 2012 or Harley 2014). Klepousniotou 2002 and Morris 2006
} 
the content words and the functional items occurring in 'A pupil was in the middle of the classroom' are linearly paired with a provisional semantic interpretation. Unambiguous words are paired with a single sense, whereas pupil is paired with both its alternative senses, and all the senses thus activated are deposited in working memory. Parallel morphosyntactic processing supervises the construction of the phrase structure for the activated senses and yields the protoproposition A [YOUNG STUDENT / EYE OPENING] WAS IN THE MIDDLE OF THE CLASSROOM. In order to associate 'A pupil was in the middle of the classroom' with a definite set of truth conditions, the speaker now needs to select one of the two candidate interpretations of pupil. To this end, a [YOUNG STUDENT / EYE OPENING] WAS IN THE MidDLE OF THE CLASSROOM is transferred into a post-semantic workspace which contrasts the statistical likelihood of A YOUNG STUDENT WAS IN THE MIDDLE OF THE CLASSROOM with that of AN EYE OPENING WAS IN THE MIDDLE OF THE CLASSROOM, pares away EYE OPENING as otiose, selects YOUNG STUDENT and delivers the truth-evaluable string A YOUNG STUDENT WAS IN THE MIDDLE OF THE CLASSROOM.

The exact nature of the selection occurring at the end of this process is not immediately relevant for our purposes: it could be a statistical inference computing on the frequency of the senses assigned to pupil in previous occasions of use, or it could involve the access to some rule-like constraint binding the interpretation of $p u-$ pil to YOUNG STUDENT whenever pupil is used in a sentence containing a relevant occurrence of classroom. Regardless of this, there is a single important point to be made for the purposes of our argument. Namely, post-semantic selection is indispensable to pair the input sentence with a definite truth-evaluable content: without the reduction of [YOUNG STUDENT / EYE OPENING] to YOUNG STUDENT, the comprehender is bound to be unable to associate 'A pupil was in the middle of the classroom' with a single set of truth conditions. Albeit I doubt that the reader has ever stepped into a classroom and spotted a bare human eye right at the center of it, there is plenty of conceivable contexts of utterance where construing pupil as EYE OPENING

both offer a nice introduction to the psychology of word sense disambiguation. Small, Cottrell, Tanenhaus 1988 is also a comprehensive, though earlier reference work on the subject. 
would make it an impeccable referential label for an object standing in the middle of a classroom (similar cases are discussed, e.g., in Searle 1980, Pelczar 2000, Recanati 2004). Simply put, there are no linguistic reasons why the interpreter should prefer YOUNG STUDENT Over EYE OPENING, and it is impossible to require that the information whereby agents select YOUNG STUDENT be part of their command of the lexico-syntactic properties of English. The discriminating factor, here, is world knowledge. ${ }^{5}$ Contra Borg, there seems to be no definite "what is said" without appealing to information outside the language faculty here, because unless the two candidates to the status of truth-evaluable content conveyed by 'A pupil was in the middle of the classroom' are tested against a background of relevant nonlinguistic knowledge, it is impossible to assign the sentence a single literal meaning. ${ }^{7}$

Now, while many accept that encoded conventional meaning is typically non-propositional and observe that in most cases disambiguation and reference determination are needed to obtain truth conditions (e.g., see Devitt 2013), semantic minimalism wants encoded

${ }^{5}$ A quick counterexample might be useful to clarify this point. The verb find is highly polysemous: it can express LOCATE, BELIEVE, REALIZE and plenty of other fine-grained senses. Suppose we need to interpret the sentence 'Mark found that the show was boring' and are asked to choose which, among LOCATE and BELIEVE, is the sense to be assigned to find. To do this, we do not need world knowledge, because it is part of our word-level command of the combinatorial properties of find that when the object slot of its argument structure is filled by a sentential complement, the verb cannot be interpreted as LOCATE (as in, e.g., 'Mark found the cat'). The situation is different in 'A pupil was in the middle of the classroom': in this case, a non-linguistic input is indispensable to perform sense selection.

${ }^{6}$ By 'what is said', I simply mean the conventional truth-conditional features that can be ascribed by a speaker $A$ to a sentence $S$ in virtue of the linguistic properties of S (hence, in virtue of A's being a competent user of the language in which $\mathrm{S}$ is expressed).

${ }^{7}$ Naturally, the example I have chosen is just one among many possible instances of homonymy, both balanced (i.e., based on word forms licensing equally dominant senses, such as cell or panel) and unbalanced (i.e., based on word forms whose alternative senses are asymmetric in frequency, such as ball or port). More precisely: in 'A pupil was in the middle of the classroom', pupil is a balanced homonym occurring in a biased sentential context, that boosts the statistical likelihood of YOUNG STUDENT. 
conventional meaning to be inherently propositional. Which makes disambiguation a problematic case. Borg (2004: 140-146; 2012: 9091, 171-172) lucidly recognizes the issue and provides some nicely argued answers regarding how it should be accommodated in the context of her minimalist proposal. In what follows, I will argue that none of such answers is entirely convincing. To be fair, I will never claim to have identified a knock-out case against Borg's thesis, but I think I can reasonably show that the best assumptions we can make about the dynamics of word sense disambiguation cast some significant doubts on the overall plausibility of minimal semantics. To proceed, let us examine how Borg suggests that her theory can accommodate cases of lexical ambiguity of the sort contemplated in 'A pupil was in the middle of the classroom'. According to Borg, minimal semantics can deal with them because disambiguation processes typically fall into one of the following cases.

(D1) Pre-Linguistic Disambiguation. Sense selection occurs before lexico-syntactic processing. Only one of the two senses of pupil is inputted to lexico-syntactic processing and only one of the two truth-evaluable contents licensed by the sentence is built.

(D2) Post-Linguistic Disambiguation. Sense selection occurs after lexico-syntactic processing. The sentence is heard as ambiguous and both its alternative truth-evaluable contents are built. After the two truth-evaluable contents have been allowed to leave the language faculty, general intelligence selects one and suppresses the other.

(D3) Linguistic Disambiguation. Sense selection occurs inside lexicosyntactic processing. This can happen in three ways.

(D3a) Both senses of the homonym are inputted to lexico-syntactic processing but only one is used to interpret the sentence, due to a habitualized preference. For example, the subject's previous encounters with the homonym have established a selectional tendency based on which her language faculty spontaneously computes one of the two senses and pares away the other. 
(D3b) Both senses of the homonym are inputted to lexico-syntactic processing but one is immediately suppressed thanks to knowledge about the preceding discourse context.

(D3c) Both senses of the homonym are inputted to lexico-syntactic processing and used to interpret the sentence. General intelligence intrudes into lexico-syntactic processing and operates as a selective inhibitor on one of the two truth-evaluable contents licensed by the sentence, before they are allowed to leave the language faculty.

\subsection{Pre-linguistic disambiguation}

Let us start with (D1): only one of the two senses of pupil is inputted to lexico-syntactic processing. Borg's proposal can be spelled out in two ways. First, preferential sense activation is direct, unconstrained and obtains independently of the general intelligence of the interpreter (let us name this hypothesis D1a). Second, preferential sense activation is the output of the early resolution of a constraintsatisfaction problem and obtains thanks to the general intelligence of the interpreter (let us name this hypothesis D1b). Opting for (D1a) would clash with a good deal of classic experimental literature on lexical access observing that in the earliest stages of language interpretations tasks, all the alternative senses of an ambiguous word are activated (regardless of which is more dominant or contextually appropriate) to be later selected via the acquaintance with semantic and non-linguistic context (e.g., Swinney 1979; Tanenhaus, Leiman, Seidenberg 1979; Seidenberg et al. 1982; Folk, Morris 2003; Mason, Just 2007). As for (D1b), the hypothesis could be reconciled with Borg's minimalism on condition that the general-purpose processes involved in pre-semantic filtering required no input from lexico-syntactic analysis, i.e., that pre-semantic filtering and lexico-syntactic analysis were two serially ordered processes among which there occurred a rigidly unidirectional interaction. Yet, it seems that in order for constrained sense activation to obtain, a rather rich flow of information from pre-semantic filtering to lexico-syntactic analysis and back from lexico-syntactic analysis to pre-semantic filtering has 
to take place. To appreciate this, let us observe that there are two essential ways in which Borg's appeal to (D1b) can be put to work. The first would be to assume that constrained sense activation is generated by a world knowledge default (\$3.1.1). The second would be to opt for a salience-first model of lexical access (§3.1.2).

\subsubsection{World knowledge defaults}

Assume that the retrieval of the senses of pupil is controlled by a world knowledge default (WKD) that constrains lexical access to the activation of YOUNG STUDENT by computing on information provided in the position occupied by classroom (easy test: were the source sentence 'A pupil was in the middle of an eye', the interpretation accessed for pupil would have been EYE OPENING) ${ }^{8}$ Now ask: is it possible for speakers to recruit the WKD and bring it to bear on the operations of the language faculty before lexico-syntactic processing has started? The answer to this question seems bound to be negative, since without some early lexico-syntactic breakdown of the sentence it is impossible for speakers to determine that the syntagmatic position occupied by classroom is the one containing the information that is relevant for the disambiguation of pupil. If it is true that subjects selectively retrieve YOUNG STUDENT because of a WKD based on a stereotypical representation of the objects they are more likely to find in classrooms, it is also true that the WKD can be brought to bear on the disambiguation of pupil only if some rudimentary construc-

\footnotetext{
${ }^{8}$ Incidentally, let it be noted that the default can be modeled with the toolkit of standard information theory. The idea is to measure the amount of information required to disambiguate among candidate interpretations for an ambiguous word in terms of entropy generated by candidate interpretations in the set of its senses, and posit that only the interpretation generating the lowest entropy will be activated. If $\mathrm{H}$ is the entropy, $\mathrm{P}$ is a measure of probability, $\mathrm{M}$ is the set of the senses of pupil, $\mathrm{C}$ is the set of contextual bits of information available in the interpretation of 'A pupil was in the middle of the classroom', $c$ is classroom, Ys is YOUNG STUDENT, and EO is EYE OPENING, then the preferential activation of YOUNG STUDENT can be predicted on grounds that $\mathrm{H}[\mathrm{YS} \mid C]<\mathrm{H}[\mathrm{EO} \mid C]$, which is to say: $-\sum_{c l \in \mathrm{C}} \mathrm{P}(c l)$ $\sum_{\mathrm{rs} \in \mathrm{M}} \mathrm{P}(\mathrm{YS} \mid c l) \log \mathrm{P}(\mathrm{YS} \mid c l)<-\sum_{c l \in \mathrm{C}} \mathrm{P}(c l) \sum_{\text {Ео } \in \mathrm{M}} \mathrm{P}(\mathrm{EO} \mid c l) \log \mathrm{P}(\mathrm{EO} \mid c l)$. More generally, classroom is a relevant bit of contextual information because $c l \in \mathrm{C}$ and $\mathrm{H}[M]$ $>\mathrm{H}[M \mid C]$, that is, $-\sum_{m \in \mathrm{M}} \mathrm{P}(m) \log \mathrm{P}(m)>-\sum_{c l \in \mathrm{C}} \mathrm{P}(c l) \sum_{m \in \mathrm{M}} \mathrm{P}(m \mid c l) \log \mathrm{P}(m \mid c l)$, with $m$ being an interpretation of pupil. See Cover, Thomas 2006.
} 
tion of the logical form of the sentence and some exploratory analysis of its lexical items have already established at least what follows: (i) that pupil is an ambiguous item describing the value of the predicate variable of an existential quantifier; (ii) that classroom is the most salient element of an optional argument signaling the location of the object labeled as pupil; (iii) that classroom "means" CLASSROOM. Unless this body of information has been processed by the lexico-syntactic parser, general intelligence is bound to be unable to determine which among the many WKDs the agent is acquainted with should be accessed and exploited for preferential sense activation. In this sense, (D1b) can be treated as a variant of (D3c), and its viability comes to depend on the possibility of reconciling Borg's minimalism with the existence of a dense informational interface between lexico-syntactic processing and general intelligence.

\subsubsection{Salience-first access}

Here the minimalist might want to observe that the psychological story we have recapitulated in $\S 2$ is not completely accurate or unbiased, since some evidence has been taken to shown that in the earliest stages of utterance processing ambiguous words do not always activate the entire range of their alternative senses, but are sometimes paired with a single "contextually salient" meaning (see, e.g., Giora 2003, 2012). If the rest of the phrase structure accepts the salient meaning (i.e., the incorporation of the salient meaning does not generate syntactic or semantic anomalies), the salient meaning is preserved and the string is allowed to leave the language faculty. Otherwise, if the rest of the phrase structure does not support the salient meaning (e.g., the salient meaning makes a predicate invalidate a selectional restriction or pushes a complement to violate the argument structure of its verb), the lexico-syntactic module activates a backtracking function which inhibits the salient meaning and recruits a more appropriate sense for the ambiguous word. Now, if we claim that the preferential activation of the salient sense is stimulated directly by the context of utterance, we face the same problem encountered in the discussion of (D1a) (i.e., in the earliest stages of language interpretations tasks, all the alternative senses of an ambiguous word are activated regardless of which is contextually appropriate). 
On the other hand, if we construe salience as a feature engendering preferential sense activation on the basis of the interpreter's previous linguistic experience (i.e., all the alternative senses of the ambiguous word are activated but only the most prominent has enough statistical strength to get to the composition phase), we face two different problems. First, by allowing that linguistic experience can have a constructive role in arranging the dominance of the senses associated to the mental representations of word forms, Borg would endorse a notion of lexical knowledge which is much more idiosyncratic and open-ended than the formalist picture of linguistic competence she aims to safeguard (2012: chapter 6). Second, it appears that the string built by lexico-syntactic processing with the dominant sense of the homonym must be verified by an additional abductive stage in order to be definitively validated. The most indicative reason for this is that backtracking can be requested even after the string built by the lexico-syntactic parser has left the linguistic module. Suppose we are dealing with a native speaker of English $\mathrm{S}$ who is informed about the ambiguity of pupil and whose previous linguistic experience is such that $\mathrm{S}$ has been prevalently exposed to uses of pupil where the word was intended to mean EYE OPENING. At some point, $\mathrm{S}$ is presented with 'A pupil was in the middle of the classroom'. S detects the word pupil, preferentially accesses EYE OPENING and integrates it in the phrase structure of the sentence. Is this sufficient to predict that $\mathrm{S}$ will validate the string constructed by interpreting pupil as EYE OPENING? Of course not. S could still evaluate that the string including EYE OPENING embeds a heuristically questionable interpretation of the sentence because the reading she has spontaneously assigned to pupil displays an insufficient degree of encyclopedic consistency with the rest of the sentential context, and activate backtracking to replace it with a less atypical interpretation. The moral is simple: when it comes to cases where the competitor interpretations for an ambiguous word generate truth-conditional strings which are equally acceptable if tested against the desiderata of lexico-syntactic well-formedness, it is impossible to require the language faculty to be responsible for the unreflective sense of implausibility a speaker accessing EYE OPENING first could feel in evaluating whether the interpretation she has assigned to our sentence is attractive. Yet, it seems that such a stage of weighted abduction is a constitutive part of the array of mental 
processes whereby cognizers assign literal content to sentences, and it is not entirely clear how this could be reconciled, to borrow Borg's (2004: 142) own words, with the somewhat "ascetic" postulation of a self-contained lexico-syntactic route to sentential meaning.

\subsection{Post-linguistic disambiguation}

Let us now turn to (D2). Borg depicts the following scenario: the sentence 'A pupil was in the middle of the classroom' is heard as ambiguous and both its alternative truth-evaluable meanings are built. After the two truth-evaluable strings have been allowed to leave the language faculty, general intelligence selects one and suppresses the other. Now, I have no complaints against the idea that disambiguation might sometimes follow this routine, but I do have some doubts regarding the compatibility between the existence of processes of post-linguistic disambiguation and Borg's minimalism. It is true that in cases of this sort lexico-syntactic calculus does a fundamental part of the job required to construct the truth-evaluable content speakers preferentially associate with the target sentence. Be it made of a single structured string with an underspecified lexical slot (A [YOUNG STUDENT / EYE OPENING] WAS IN THE MIDDLE OF THE CLASSROOM), be it made of two fully truth-evaluable strings to be evaluated and selected as wholes (A YOUNG STUDENT WAS IN THE MIDDLE OF THE CLASSROOM vs. AN EYE OPENING WAS IN THE MIDDLE OF THE CLASSROOM), the acquaintance with the lexico-syntactic features of our sentence yields some semantically informative content. Yet, by dropping the tenet that the truth-evaluable content of well-formed declarative sentences is always and entirely dictated by lexico-syntactic processing, minimalism would dispose of the key claim thanks to which it promised to offer an interesting and controversial insight on language processing in the first place. The point of Borg's minimalism is not to merely suggest that lexico-syntactic calculus and linguistic knowledge have a crucial and modularly defined role in the recovery of sentential meaning, or that they are "usually" sufficient to obtain truth-evaluable content. The point of Borg's minimalism (e.g., 2012: 48) is to propose that lexico-syntactic calculus and linguistic competence are all it takes to build semantically complete content, and it is difficult not to remain skeptical about how the case we have discussed is sup- 
posed to meet this Davidsonian desideratum. The very choice of labeling comparable processes of disambiguation as 'post-semantic' looks unwarranted. Granted, they are supposed to intervene on the output of the Fodorian module Borg assigns to lexico-syntactic processing and, in this sense, their temporal niche naturally falls in the postmodular inferential phase. But if it is true that they are indispensable to generate (rather than modulate or enrich) the literal content of the sentence in need of disambiguation, is it still fair to characterize them as something bona fide "post-semantic"?

\subsection{Linguistic disambiguation}

Finally, let us turn to (D3). Borg suggests that it is possible to locate disambiguation within lexico-syntactic processing while preserving the minimalist framework in three scenarios, respectively corresponding to (D3a), (D3b) and (D3c). In the first case, both the senses of pupil are inputted to lexico-syntactic processing but only one is used to interpret the sentence, due to a habitualized preference. In the second case, both the senses of pupil are inputted to lexico-syntactic processing, but one is rapidly suppressed thanks to information acquired from the preceding discourse context. In the third case, both the senses of pupil are inputted to lexico-syntactic processing and used to interpret the sentence, but general intelligence acts as a selective inhibitor on one of the two truth-evaluable contents licensed by the sentence while they are being built.

As for (D3a), it seems that adhering to this line of reply would again relax the notion of 'linguistic knowledge' beyond the limits tolerated by Borg's commitment to a formalist understanding of natural language semantics. As we observed in $\S 3.1 .2$, by accepting that factors such as conventionality, distributional frequency and familiarity can affect the offline dominance of the senses associated to word forms in the mental lexicon, the minimalist would endorse a view of lexical competence which is much more "pragmatic" than the one its intellectualist understanding of semantic competence should aim to sustain. More precisely, if the representational repertoire underpinning our ability to make competent use of word forms contained instructions of type '(when embedded in the semantic context C) the word $w$ preferentially takes the sense $m$ ', word knowledge would 
more closely mirror the abductively rich notion of lexical semantic knowledge adopted by cognitive linguists (e.g., Evans 2009) and by most NLP approaches to word sense disambiguation (e.g., Manning, Schütze 1999; Agirre, Edmonds 2006), rather than the axiomaticlogicistic picture of lexical meaning envisaged by Borg (e.g., 2010).

As for (D3b) and (D3c), I suspect that allowing linguistic performance to be inhibited by the discourse context would jeopardize Borg's appeal to a modularist account of lexico-syntactic processing. Borg (2004: 93, 144; 2012: 64) is right in observing that allowing information about the discourse context to play a role as a selective top-down filter on meaning construction processes is very different from making room for the kind of all-pervasive constructive role for non-linguistic context envisaged by some contextualists. Even so, the "intrusion" licensed by Borg seems far from innocent here: however weakly one chooses to interpret it, it remains based on the assumption that the core computations of the lexico-syntactic module can be stopped by the short-term representations in which the interpreter has stored the background information she has acquired from the discourse context. This would be absolutely fine in a pragmatically-oriented approach such as discourse representation theory (Kamp, Reyle 1993), segmented discourse representation theory (Asher, Lascarides 2003), or file change semantics (Heim 1988), but seems to clash with Borg's appeal to a full-fledged Fodorian picture of lexico-syntactic processing (which includes the mandatoriness requirement: in order for some system to constitute a module, its operations must run to completion every time they are switched on by presentation of a relevant stimulus; see Fodor 1983). ${ }^{9}$ Hence, even if the susceptibility to inhibition displayed by linguistic performance

\footnotetext{
${ }^{9}$ Other studies support the same worry. For example, Bicknell et al. 2010 and Matsuki et al. 2011 report an increase in reading times for sentences in which an agent-verb combination is followed by a statistically incongruent (though linguistically plausible) patient (e.g., 'The journalist checked the spelling of his latest report' vs. 'The mechanic checked the spelling of his latest report'). The immediacy of this slowdown would seem to require either that world knowledge must be embedded in the lexicon, or else that world knowledge can affect the amount of time required to carry out the analysis of the linguistic properties of a sentence by manipulating the operations of the lexico-syntactic parser while they are being performed. Both options look problematic for minimal semantics.
} 
in word sense disambiguation were as selective as Borg believes it is, this could still be sufficient to conclude, contra the minimalist, that lexico-syntactic processing should be better characterized as a weakly modular system, i.e., domain-specific and designed to contribute to the construction of truth-evaluable content by interfacing its operations with information exceeding the knowledge base of the lexico-syntactic parser. Ironically enough, the very idea Borg is so concerned with rejecting (i.e., broadening the data base of linguistic processing so as to make it compute information outside the lexicosyntactic province) might be the best way to safeguard the minimalist thesis that lexico-syntactic processing is systematically sufficient to assign sentences a truth-evaluable content via a fully modular information processing routine.

\section{Multiplying types}

I add to the list of possible responses an answer that Borg does not consider explicitly, but that I find worth articulating and discussing. Let me express it in the form of a question: why not conceive A YOUNG STUDENT WAS IN THE MIDDLE OF THE CLASSROOM and AN EYE OPENING WAS IN THE MIDDLE OF THE CLASSROOM as the (minimal) propositions expressed by two different sentence-types whose respective English instantiations happen to be phonographically indiscernible? After all, homonymy is standardly understood as an $n$-ary relation between different terms that share the same spelling and pronunciation (e.g., Murphy 2010). So it should stand to reason to argue that the possibility to pair our sentence with two truth-evaluable contents arises because 'The pupil was in the middle of the classroom' is the realization of two sentence-types that in English happen to be expressed through utterances and inscriptions sharing the same surface properties. The argument could run as follows: (i) the distinction between A YOUNG STUDENT WAS IN THE MIDDLE OF THE CLASSROOM and AN EYE OPENING WAS IN THE MIDDLE OF THE CLASSROOM corresponds to the distinction between two sentence-types, $\alpha$ and $\beta$; (ii) the logical forms of $\alpha$ and $\beta$ terminate in the subject position with different NPs ( $\alpha$ hosts the constituent pupil $_{\alpha}$, while $\beta$ hosts the constituent pupil ${ }_{\beta}$ ); (iii) since pupil $\alpha$ and pupil $\beta$ are homonyms in English, the difference between the sets of lexical types respectively hosted by $\alpha$ and $\beta$ can- 
not be expressed in the surface realization of $\alpha$ and $\beta$; (iv) based on (i-iii), disambiguating pupil actually amounts to determining whether we should process $\alpha$ or $\beta$; (v) once the relevant sentence-type ( $\alpha$ or $\beta$ ) has been selected, its logical form can be recovered via the information processing routine evoked by the minimalist. The argument looks viable and the minimalist can endorse it, perhaps reinforcing it via psycholinguistic claims about homonyms' having a separate mental representation for each of their alternative senses (see, e.g., Frazier, Rayner 1990; Klein, Murphy 2001; Beretta, Fiorentino, Poeppel 2005; Brown 2008), and via a suitably strengthened version of the hidden homonymy approach to color terms ambiguity argued by Kennedy and McNally (2010), a line of argument Borg herself (2012: 91) is sympathetic with. The problem is that since the English instantiations of $\alpha$ and $\beta$ are indiscernible and therefore bound to be perceived as a single phonographic input, the theoretical distinction between the two sentence-types cannot be used to increase the plausibility of Borg's account of the psychology of language processing. As the disambiguation between $\alpha$ and $\beta$ cannot be performed on the basis of phonological, presuppositional, graphic or intonational variables, ceteris paribus their presentation is bound to be perceived as the presentation of the same sentence and to trigger the same cognitive responses across occasions of interpretation.

To have a clearer grasp of the problem, think of quantifier raising in structurally ambiguous sentences such as 'Some boy loves every girl' (let us name this sentence S). Here we have one surface form admitting two logical forms (LFs), depending on which of the two quantifiers is assigned the wider scope $(\exists>\forall$ vs. $\forall>\exists)$. In this case, the argument from the minimalist side could run as follows. Since every well-formed sentence-type is paired with just one LF, the possibility to dislocate the quantifiers of $S$ from their surface position to their scope position in two ways proves that $S$ is the surface realization of two sentence-types. Accordingly, the assumption of a purely lexico-syntactic route to sentential meaning can be preserved on grounds that it cannot be the case that the cognitive processes involved in the treatment of the two types are the same: they are distinct and each consistent with the minimalist view of sentence pro- 
cessing. ${ }^{10}$ Once more, the reply looks viable, but it seems it underestimates an important detail: unless some independent input as to which of the two quantifier dislocations should be favored is given, it is impossible to determine the literal content conveyed by $\mathrm{S}$ (qua phonographic input) by relying only on the lexical and syntactic features that are manifested at the surface level. It is more than reasonable to expect the cognitive processes involved in the construction of its two interpretations to be different, but neither of the two can be preferentially triggered if context does not provide some disambiguation clue signaling which of the two types fits the conversational setting. Which means, in turn, that the minimalist can count on this line of reply on condition that lexico-syntactic processing operates directly on disambiguated sentence-types rather than on surface forms, thereby evading the question of how (if not via context-sensitive lexico-syntactic processing) we identify sentence-types and evaluate their contextual plausibility in the first place. Borg might wish to insist that the case is easy to accommodate within her proposal, since she agrees that non-linguistic information can play a role in letting context help select one of the two sentence-types via the selection of its LF. If this were the case, Borg would still fall short of her aspiration to offer a realistic explanation of meaning recovery: in order to get to truth-evaluable content, you must pass through LF selection, and lexico-syntactic processing alone will not do it. In other words, if semantic minimalism wants to live up to its psychological ambitions and give us a plausible story about how agents competent in a linguistic idiom come to grasp the meaning of sentences expressed in that idiom, it should tell us something about how we go from being exposed to unprocessed collections of phonographic events to the entertainment of truth-evaluable thoughts. Unfortunately, it is difficult to tell how narrowing the framework down to an account of the processes governing the assignation of truth conditions to abstract LFs could produce a cognitively instructive account of semantic performance (or even of a stage of semantic performance). ${ }^{11}$

${ }^{10}$ I am grateful to Emma Borg for pressing me to address this point.

${ }^{11}$ In addition, it should perhaps be observed that while the low semantic overlap characterizing the alternative meanings of a homonym is consistent with an appeal to the distinction between lexical types, the same strategy is unlikely to 
Very crudely put, it seems that the minimalist has to choose sides. Either she restricts herself to arguing that her claims are intended to spell out the deductive aspects of the semantics of sentence-types and that her emphasis on 'meaning recovery' should be interpreted as a philosophically informed rationalization of the analytical routine whereby orthodox semanticists look into the truth-evaluable content of logically transparent types, or she bets on the hypothesis that her framework can be exploited to describe the mental processes whereby ordinary speakers assign literal content to sentences in concrete and situated events of language use. If the latter option is the case, then my (admittedly epistemological) worry is that if minimalism does not aim to contemplate in some interesting fashion all that is involved in the extraction of semantic information from surface forms, its appeal to a "purely lexico-syntactic route" to truth-evaluable content runs the risk of insisting on a notion of linguistic processing which has nothing to do with the way we use natural languages.

Part of this plea for empirical responsibility is insightfully recommended by Borg, who adds that although minimalism is open to be disconfirmed by the psychological evidence, such evidence must be about speakers' knowledge of their language, not about the psychological processes whereby speakers assign content to sentences (Borg 2012: 64). Yet, even this caveat looks problematic if measured against the rest of Borg's claims. First, it is generally well-established that linguistic knowledge and the dynamics of utterance processing cannot be kept too separate, since any plausible theory of the organization of linguistic knowledge is bound to imply rather specific claims about the way such knowledge operates in language comprehension and production, and any plausible theory of the cognitive processes underlying language use is bound to imply rather specific claims about the organization of linguistic knowledge. ${ }^{12}$ But more importantly, if

result pursuable in cases of ambiguity generated by word forms whose possible senses are significantly more related. For example, it would be more controversial to postulate a separate lexical type for each of the different senses of a polysemous verb like take. Yet, it seems to me that many of the objections this paper has raised against minimal semantics by focusing on the disambiguation of homonymy could be formulated just as fittingly by considering the processing of polysemy. For issues of space, I cannot elaborate further on this point.

${ }^{12}$ For example, the psycholinguistic research on the mental lexicon is stan- 
the only type of evidence that can disconfirm Borg's minimalism is about linguistic knowledge (which is by definition a matter of linguistic competence), does this mean that Borg's proposal should be evaluated exclusively with respect to its ability to account for linguistic competence? In such a case, even if we agreed that minimalism is the best deal on the table to make sense of speakers' command of their language, it is not immediately clear why the endorsement of a minimalist view of lexico-syntactic competence should ipso facto be interested in trading in the possibility to pair well-formed declarative sentences with truth-evaluable content just through lexicon and syntax. That we should have a minimalist view of lexico-syntactic knowledge does not necessarily mean that we should have a minimalist theory of lexico-syntactic performance: the two are very different animals. The same problem seems to undermine Borg's vindication of the minimalist thesis as the natural inheritor of a modularist view of lexico-syntactic processing (e.g., 2012: 13). That we should have a modularist picture of lexico-syntactic processing does not necessarily mean that we should have a modularist view of the entire range of processes involved in the recovery of propositional meaning: lexicosyntactic processing might well satisfy the strong modularity constraint and yet be insufficient to generate truth-evaluable content. For example, one could easily endorse a Fodorian picture of lexicosyntactic processing while maintaining that all the language faculty can do with a well-formed declarative sentence $\mathrm{S}$ is to pair $\mathrm{S}$ with an abstract template that relates to the truth-evaluable content eventually selected for $S$ just as character relates to content in the variety of contextualism popularized by Kaplan.

\section{Concluding remarks}

To sum up. I have reviewed the main tenets on Borg's framework and clarified the way the disambiguation of homonymy poses a challenge to the account of language processing proposed by semantic minimalism. I have then examined the solutions offered by Borg in order to accommodate the problem, and argued that none of them

dardly understood as an attempt to infer claims about the nature of lexical representations from the study of lexical activity. See, e.g., Jarema, Libben 2007. 
offers a fully convincing argument to embrace the austere picture of meaning recovery recommended by semantic minimalism against accounts of linguistic processing where the composition of truthconditional meaning is vastly context-dependent and richly interfaced with world knowledge and general purpose abilities. ${ }^{13}$ Now let me conclude with a couple more constructive proposals. First, I suggest that Borg should opt for a more nuanced picture of the limitations of lexico-syntactic processing and follow the recommendation already argued, among others, by Bach (2007): do without propositionalism. Which means, give up on the claim that every well-formed (indexical-free) declarative sentence expresses a truthevaluable content which is fully determined by its lexico-syntactic features and recoverable with no need to access non-linguistic information, and make room for the notion of a 'propositional radical' (or for any other construct doing the same explanatory work carried out by propositional radicals, e.g., the schematic truth-conditional templates of relevance theorists). In Borg's (2012: 208) terms: make room for the talk of incomplete logical forms and move toward the "perilous fine line" separating radical minimalism from contextualism. Hence, allow that in some cases the best the acquaintance with the overt lexico-syntactic properties of a sentence can do is pair it with a truth-conditionally incomplete content, while denying that filling the gap required to turn it into a genuine truth-evaluable content has to be based on linguistic knowledge or to be classified as a semantic matter. Second, Borg's minimalism may be much better off as an account of linguistic competence. To my understanding, it is not only possible, but perhaps even desirable to pair a modularly inspired understanding of lexico-syntactic knowledge with the view that the recovery of truth-evaluable content is a phenomenon which is designed to arise via the cooperation of linguistic and non-linguistic information. One simple way to do this could be to introduce a

${ }^{13}$ Although in this article I have restricted myself to questioning Borg's minimalism, the points I have tried to make should apply equally well to Cappelen and Lepore's (2005) minimalism. The reason why I have chosen to confine my discussion to Borg's work is simple. Semantic minimalism owes us an account of its empirical plausibility, but while Cappelen and Lepore tend to shy away from that task, Borg is eager to explore the psychological tenability of her assumptions. Which makes her work a more suitable contact point for my arguments. 
distinction between 'knowledge modules' and 'processing modules' (Coltheart 1999), and bet on the research hypothesis that lexicosyntactic analysis is based on weakly modular (i.e., domain-specific and interactive) processor which computes on information retrieved from a strongly modular knowledge base, corresponding to what is standardly referred to as speakers' "knowledge of the language".

After all, if there is one lesson we can plausibly learn from the very existence of ambiguity in natural languages, that lesson is precisely that language processing is designed to interact with general purpose abilities. Functionalist accounts of ambiguity have long been arguing that the presence of ambiguity in natural languages should prove that the language faculty has not evolved for purposes of communication, since, if that were the case, linguistic forms would map bijectively to meanings, and comprehenders would not need to expend effort in inferring literal meaning or speech act content via non-linguistic information (e.g., Pinker, Bloom 1990; Chomsky 2002). On the contrary, it can be argued that ambiguity is in fact a desirable property of communication codes, because it allows for linguistic systems which are formally parsimonious, optimized for memorization and exploitable by drawing computational resources from different domains of cognition. As Piantadosi, Tily and Gibson (2012) have noted, there are two important facts that make ambiguity a desirable feature of linguistic systems and support the intuition that ambiguity results from a pressure for efficient communication. First, when sentential or extra-linguistic context is informative about meaning, a completely unambiguous language would become partly redundant with context and therefore inefficient (no wonder that homonyms and highly polysemous words preserve their ambiguity in a strikingly small proportion of the cases in which they are embedded in a sentence or used in a speech act). Second, by mapping phonographic units to multiple meanings, ambiguity reduces the number and the length of word forms that have to be stored in the mental lexicon to secure efficient linguistic performance, maintains our internal word store at a low degree of phonotactical complexity, and allows language users to speed up the transmission of information via speech despite the physiological limitations of their articulatory apparatus (e.g., Levinson 2000). ${ }^{14}$

${ }^{14}$ Many thanks to Emma Borg, François Recanati, Diego Marconi and Ja- 


\author{
Luca Gasparri \\ Institut Jean Nicod, ENS Paris \\ UMR 8129, Pavillon Jardin \\ 29 rue d’Ulm, 75005 Paris, France \\ luca.gasparri@ens.fr
}

\title{
References
}

Agirre, Eneko, and Edmonds, Philip. Eds. 2006. Word Sense Disambiguation: Algorithms and Applications. Berlin: Springer.

Asher, Nicholas and Lascarides, Alex. 2003. Logics of Conversation. Cambridge: Cambridge University Press.

Bach, Kent. 2007. The Excluded Middle. Semantic Minimalism Without Minimal Propositions. Philosophy and Phenomenological Research 53: 435-442.

Beaver, David. 2001. Presupposition and Assertion in Dynamic Semantics. Stanford, CA: CSLI Publications.

Beretta, Alan, Fiorentino, Robert and Poeppel, David. 2005. The Effects of Homonymy and Polysemy on Lexical Access: An MEG Study. Cognitive Brain Research 24: 57-65.

Bicknell, Klinton, Elman, Jeffrey L., Hare, Mary, McRae, Ken and Kutas, Marta. 2010. Effects of Event Knowledge in Processing Verbal Arguments. Journal of Memory \& Language 63: 489-505.

Borg, Emma. 2004. Minimal Semantics. Oxford: Oxford University Press.

Borg, Emma. 2007. Minimalism versus Contextualism in Semantics. In ContextSensitivity and Semantic Minimalism: New Essays on Semantics and Pragmatics. Edited by Gerhard Preyer and Georg Peter. New York, NY: Oxford University Press.

Borg, Emma. 2009. Semantic Minimalism. In The Routledge Pragmatics Encyclopedia, edited by Louise Cummings. London: Routledge.

Borg, Emma. 2010. Minimalism and the Content of the Lexicon. In Meaning and Context. Edited by Luca Baptista and Erich Rach. Bern: Peter Lang.

Borg, Emma. 2012. Pursuing Meaning. Oxford: Oxford University Press.

Brown, Susan W. 2008. Polysemy in the Mental Lexicon. Colorado Research in Linguistics 21: 1-12.

Cappelen, Herman and Lepore, Ernest. 2005. Insensitive Semantics: A Defense of

copo Tagliabue for valuable input on an earlier draft of the paper. I also thank two anonymous reviewers for their comments on the submitted manuscript. The usual disclaimer applies. Part of the research that led to this paper has received funding from the European Union's Seventh Framework Program (FP7/20072013, MSCA-COFUND) under grant agreement No. 245743, Post-Doctoral Program Braudel-IFER-FMSH, in collaboration with the Institut Jean Nicod and the Labex IEC, ENS Paris. 
Semantic Minimalism and Speech Act Pluralism. London: Wiley.

Chomsky, Noam. 2002. On Nature and Language. Cambridge: Cambridge University Press.

Coltheart, Max. 1999. Modularity and Cognition. Trends in Cognitive Sciences 3: 115-120.

Cover, Thomas M. and Thomas, Joy A. 2006. Elements of Information Theory. $2^{\text {nd }}$ edn. London: Wiley.

Davis, Wayne A. 2013. On Nonindexical Contextualism. Philosophical Studies 163: 561-574.

Devitt, Michael. 2013. Is There a Place for Truth-Conditional Pragmatics? Teorema XXXII/2: 85-102.

Evans, Vyvyan. 2009. How Words Mean: Lexical Concepts, Cognitive Models, and Meaning Construction. Oxford: Oxford University Press.

Fodor, Jerry A. 1983. The Modularity of Mind. Cambridge, MA: MIT Press.

Folk, Jocelyn R. and Morris, Robin K. 2003. Effects of Syntactic Category Assignment on Lexical Ambiguity Resolution in Reading: An Eye Movement Analysis. Memory \& Cognition 31: 87-99.

Frazier, Lyn and Rayner, Keith. 1990. Taking on Semantic Commitments: Processing Multiple Meanings vs. Multiple Senses. Journal of Memory and Language 29: 181-200.

Giora, Rachel. 2003. On Our Mind: Salience, Context, and Figurative Language. New York, NY: Oxford University Press.

Giora, Rachel. 2012. The Psychology of Utterance Processing: Context vs Salience. In The Cambridge Handbook of Pragmatics. Edited by Kasia M. Jaszczolt and Keith Allan. Cambridge: Cambridge University Press.

Harley, Trevor A. 2014. The Psychology of Language: From Data to Theory, $4^{\text {th }} \mathrm{edn}$. New York, NY: Psychology Press.

Heim, Irene. 1983. On the Projection Problem for Presuppositions. In Second Annual West Coast Conference on Formal Linguistics. Edited by Dan Flickinger and Michael Westcoat. Stanford, CA: Stanford University Press.

Heim, Irene. 1988. The Semantics of Definite and Indefinite Noun Phrases. New York, NY: Garland.

Jarema, Gonia and Libben, Gary. Eds. 2007. The Mental Lexicon: Core Perspectives. Amsterdam: Elsevier.

Jaszczolt, Kasia M. 2007. On Being Post-Gricean. In Interpreting Utterances: Pragmatics and Its Interfaces. Edited by Randi A. Nilsen, Nana A. A. Amfo and Kaja Borthen. Oslo: Novus.

Kamp, Hans and Reyle, Uwe. 1993. From Discourse to Logic. Dordrecht: Kluwer. Kennedy, Christopher. 2007. Vagueness and Grammar: The Semantics of Relative and Absolute Gradable Adjectives. Linguistics and Philosophy 30: 1-45.

Kennedy, Christopher and McNally, Louise. 2010. Color, Context and Compositionality. Synthese 174: 79-98.

Klein, Devorah E. and Murphy, Gregory L. 2001. The Representation of Polysemous Words. Journal of Memory and Language 45: 259-282.

Klepousniotou, Ekaterini. 2002. The Processing of Lexical Ambiguity: 
Homonymy and Polysemy in the Mental Lexicon. Brain and Language 81: 205-223.

Korta, Kepa and Perry, John. 2006. Varieties of Minimalist Semantics. Philosophy and Phenomenological Research 73: 451-459.

Levinson, Stephen J. 2000. Presumptive Meanings: The Theory of Generalized Conversational Implicature. Cambridge, MA: MIT Press.

MacFarlane, John. 2007. Semantic Minimalism and Nonindexical Contextualism. In Context-Sensitivity and Semantic Minimalism: New Essays on Semantics and Pragmatics. Edited by Gerhard Preyer and Georg Peter. New York, NY: Oxford University Press.

MacFarlane, John. 2009. Nonindexical Contextualism. Synthese 166: 231-250.

Manning, Christopher D. and Schütze, Hinrich. 1999. Foundations of Statistical Natural Language Processing. Cambridge, MA: MIT Press.

Mason, Robert A. and Just, Marcel A. 2007. Lexical Ambiguity in Sentence Comprehension. Brain Research 1146: 115-127.

Matsuki, Kazunaga, Chow, Tracy, Hare, Mary, Elman, Jeffrey L., Scheepers, Christoph and McRae, Ken. 2011. Event-based Plausibility Immediately Influences On-Line Language Comprehension. Journal of Experimental Psychology: Learning, Memory, \& Cognition 37: 913-934.

Morris, Robin K. 2006. Lexical Processing and Sentence Context Effects. In Handbook of Psycholinguistics. Edited by Matthew J. Traxler and Morton. A. Gernsbacher, $2^{\text {nd }}$ edn. San Diego, CA: Academic Press.

Murphy, M. Lynne. 2010. Lexical Meaning. Cambridge: Cambridge University Press.

Pelczar, Michael. 2000. Wittgensteinian Semantics. Noûs 34: 483-516.

Piantadosi, Steven T., Tily, Harry and Gibson, Edward. 2012. The Communicative Function of Ambiguity in Language. Cognition 122: 280-291.

Pinker, Steven and Bloom, Paul. 1990. Natural Language and Natural Selection. Behavioral and Brain Sciences 13: 707-784.

Recanati, François. 2004. Literal Meaning. Cambridge: Cambridge University Press. Searle, John. 1980. The Background of Meaning. In Speech Act Theory and Pragmatics. Edited by John Searle, Ferenc Kiefer and Manfred Bierwisch. Dordrecht: Reidel.

Seidenberg, Mark S., Tanenhaus, Michael K., Leiman, James M. and Bienkowski, Marie. 1982. Automatic Access of the Meanings of Ambiguous Words in Context: Some Limitations of Knowledge-Based Processing. Cognitive Psychology 14: 489-573.

Small, Steven, Cottrell, Garrison, and Tanenhaus, Michael K. Eds. 1988. Lexical Ambiguity Resolution: Perspectives from Psycholinguistics, Neuropsychology, and Artificial Intelligence. San Mateo, CA: Morgan Kaufman.

Spivey, Michael, McRae, Ken, and Joanisse, Marc. Eds. 2012. The Cambridge Handbook of Psycholinguistics. Cambridge: Cambridge University Press.

Stojanovic, Isidora. 2008. The Scope and the Subtleties of the Contextualism/ Literalism/Relativism Debate. Language and Linguistics Compass 2: 1171-1188.

Swinney, David A. 1979. Lexical Access During Sentence Comprehension: (Re) Consideration of Context Effects. Journal of Verbal Learning and Verbal Behavior 
18: 645-659

Tanenhaus, Michael K., Leiman, James and Seidenberg, Mark. 1979. Evidence for Multiple Stages in the Processing of Ambiguous Words in Syntactic Contexts. Journal of Verbal Learning and Verbal Behavior 18: 427-440.

Traxler, Matthew J. 2011. Introduction to Psycholinguistics: Understanding Language Science. London: Wiley-Blackwell.

Van der Sandt, Rob. 1992. Presupposition Projection as Anaphora Resolution. Journal of Semantics 9: 333-377. 